rating of $3.5 / 5) ; 47 \%$ of them received no ratings. There was no relationship between download frequency and rating; the most downloaded app (10k-50k downloads) received 20 reviews.

Conclusion Our study indicates that as yet, there are no fully functional apps that support the user throughout the entire pathway of STI awareness, testing, diagnosis management, prescription, partner notification and health promotion. There is a pressing need for sexual health apps which are validated and certified based on reliable content and meet high operability, privacy and security standards to appropriately exploit the potential health care benefits of mobile sexual health.

\section{P5.059 DEVELOPIMENT OF A SMALL-MEDIA INTERVENTION TO BOOST HEALTHCARE PROVIDERS' KNOWLEDGE AND AWARENESS OF SYPHILIS IN AN URBAN US COMMUNITY}

doi:10.1136/sextrans-2013-051184.1103

'B P Stoner, ${ }^{1} \mathrm{~K}$ E Holzhauer, ${ }^{2} \mathrm{C}$ G Johnson, ${ }^{3} \mathrm{C}$ Highfill. ${ }^{1}$ Washington University in St. Louis, St. Louis, MO, United States; ${ }^{2}$ St. Louis Effort for AIDS, St. Louis, MO, United States; ${ }^{3}$ Missouri Department of Health, St. Louis, MO, United States

Background St. Louis, Missouri remains a high-morbidity syphilis community. Implementation of a small-media intervention in 2005 to improve provider knowledge and awareness of syphilis led to transient declines in infectious syphilis, but rates have increased in recent years. We describe a formative collaborative project between university, health department, and community service organisation workers to develop a pocket-sized visual aid for boosting healthcare providers' awareness and understanding of syphilis epidemiology, clinical presentation, and treatment recommendations.

Methods In 2012, physician and nurse-practitioner key informants $(\mathrm{N}=18)$ provided foundational data on essential areas of provider knowledge deficit, including (1) clinical aspects of syphilis management such as lesion characteristics, diagnostic tests, and treatment approaches; (2) epidemiological aspects such as populations affected and distribution by age, race/ethnicity, and gender; and (3) health department linkages for partner referral and evaluation. Best practises for reaching providers were also considered, including mailbased and internet-based modalities

Results Several important aspects of current syphilis clinical presentation and epidemiological patterns were underappreciated by local healthcare providers, including high rates of syphilis among men who have sex with men (MSM), as well as high rates of syphilis-HIV co-infection. Rectal and oropharyngeal infection were also underrecognized. All of these were new developments since the previous version of small-media intervention. Informant recommendations led to the development of a high-contrast pocketsize small-media pamphlet containing text as well as visual prompts to boost provider knowledge and awareness of syphilis. The booster intervention is currently being rolled out city-wide through direct-mail and in-person distribution to primary healthcare providers

Conclusion Changing syphilis epidemiology requires continued vigilance among provider groups tasked with early identification, treatment, and referral of populations at risk. Small-media visual aids to boost provider awareness and knowledge of syphilis transmission patterns are an important component of larger communitylevel syphilis prevention agendas.

\section{P5.060 COMPARISON OF THE APTIMA HIV-1 QUANT ASSAY TO THE COBAS AMPLIPREP/COBAS TAQMAN HIV-1 TEST, V2.0}

doi:10.1136/sextrans-2013-051184.1104

A Hatzakis, H Papachristou, M Kantzanou. Dept of Hygiene, Epidemiology \& Medical Statistics, University of Athens Medical School, Athens, Greece
Background The APTIMA HIV-1 Quant Assay is a fully automated quantitative assay being developed on the PANTHER system and based on real-time Transcription-Mediated Amplification technology. This assay is intended for monitoring HIV-1 viral load in plasma specimens using a $0.5 \mathrm{~mL}$ sample.

Methods A cohort of 245 clinical specimens from University of Athens Medical School was tested using the COBAS AmpliPrep/ COBAS TaqMan HIV-1 Test, v2.0 (Roche Assay) and the APTIMA HIV-1 Quant Assay (APTIMA Assay). The specimens included subtypes $A, B, C$ and $G$ as well as circulating recombinant forms of HIV-1.

Results Using a lower limit of quantitation for the APTIMA Assay of 30 copies $/ \mathrm{mL}, 175$ specimens gave results quantifiable for both assays. The correlation between the two assays was excellent (0.98), with a slope of 1.06 and an intercept of -0.13 . Sixty-nine specimens gave results that were either detectable but not quantifiable or not detectable in at least one assay. Thirty were not detectable in both assays and 14 were detectable in both assays. The APTIMA Assay detected 13 specimens that were undetectable with the Roche Assay. There were 12 specimens that were detectable with the Roche Assay and undetectable with the APTIMA Assay. One specimen was above the upper limit of quantitation (10,000,000 copies/mL).

Conclusion The APTIMA HIV-1 Quant Assay gave comparable viral load results when compared to the Roche COBAS AmpliPrep/ COBAS TaqMan HIV-1 Test, v2.0. The sensitivity of the APTIMA HIV-1 Quant Assay is similar to that of the Roche COBAS AmpliPrep/COBAS TaqMan HIV-1 Test, v2.0.

\section{P5.061 MICROWAVE-ACCELERATED METAL-ENHANCED FLUORESCENCE (MAMEF) POINT-OF-CARE TEST FOR THE DETECTION OF CHLAMYDIA TRACHOMATIS}

doi:10.1136/sextrans-2013-051184.1105

'J H Melendez, ${ }^{2} \mathrm{~J}$ Huppert, ${ }^{2} \mathrm{E}$ Hesse, ${ }^{3} \mathrm{M}$ Jett-Goheen, ${ }^{3} \mathrm{~N}$ Quinn, ${ }^{3} \mathrm{C}$ A Gaydos, ${ }^{1} \mathrm{C}$ D Geddes. IInstitute of Fluorescence UMBC, Baltimore, MD, United States; ${ }^{2}$ Cincinnati Children's Hospital Medical Center, Cincinnati, $\mathrm{OH}$, United States; '3Johns Hopkins University School of Medicine, Baltimore, MD, United States

Background Chlamydia trachomatis (CT) is the most prevalent bacterial sexually transmitted infection (STI) reported worldwide. Accurate point-of-care diagnostic tests are urgently needed for the rapid treatment of patients. To address this need, we have previously developed a 16S rRNA-based Microwave-Accelerated MetalEnhanced Fluorescence (MAMEF) assay for the detection of CT. Here we report the development of an additional CT cryptic plasmid-based MAMEF assay, the use of the assays on clinical samples and the implication of MAMEF as a point-of-care test.

Methods The cryptic plasmid-based assay was investigated with cultured, titered CT and vaginal specimens. Following the optimization of the assay, we tested a blinded cohort of dry-shipped vaginal swabs using both the 16S rRNA- and cryptic plasmid-based MAMEF assays, and compared the results to nucleic acid amplification tests (NAATs).

Results The MAMEF assays detected as few as $10 \mathrm{IFU} / \mathrm{mL}$ of CT in less than 10 minutes including DNA extraction and detection. A total of 257 vaginal swabs from 245 adolescent women (ages 14-22) were analysed by MAMEF. The overall prevalence of CT by NAAT was $17.5 \%$. Of the 45 NAAT CT-positive samples and 212 CT-negative samples, 33/45 and 197/212 were correctly identified by both MAMEF assays (sens $73.3 \%$, spec $92.9 \%$ ). Using the plasmid-based assay alone, 37/45 CT+ and 197/212 CT- were detected (sens $82.2 \%$; spec $92.9 \%$ ). Using the $16 \mathrm{~S}$ rRNA assay alone, 34/45 CT+ and $197 / 212$ CT- (sens $75.5 \%$; spec 92.9 ). For the overall \% agreement with NAAT, the individual $16 \mathrm{~S}$ rRNA and cryptic plasmid were $89.8 \%$ and $91 \%$, respectively. 
Conclusions Both CT MAMEF assays demonstrated moderate sensitivity and high specificity when using dry-shipped vaginal swabs. The cryptic plasmid assay was more sensitive than the $16 \mathrm{~S}$ rRNA assay. MAMEF is an ultra-rapid platform with results in less than 10 minutes making it ideal as a point-of-care test.

\section{P5.062 AN EASY TO USE REAL TIME PCR TEST FOR CHLAMYDIA TRACHOMATIS AND NEISSERIA GONORRHOEAE PROVIDING RESULTS THAT CAN GUIDE TREATMENT CHOICES BEFORE THE PATIENT LEAVES THE CLINIC}

doi:10.1136/sextrans-2013-051184.1106

'C A Gaydos, ${ }^{2 B}$ Van Der Pol, ' $\mathrm{M}$ Jett-Goheen, ${ }^{1} \mathrm{M}$ Barnes, ${ }^{1} \mathrm{~N}$ Quinn, ${ }^{3} \mathrm{C}$ Clark, ${ }^{3} \mathrm{G} \mathrm{E}$ Daniel, ${ }^{3 P}$ B Dixon, E W Hook, '3II. 'Johns Hopkins University, Baltimore, MD, United States: 2Indiana Univ School of Public Health \& School of Med, Bloomington and Indianapolis, IN, United States; ${ }^{3}$ University of Alabama at Birmingham, Birmingham, AL, United States

Background New diagnostics for Chlamydia trachomatis (CT) and Neisseria gonorrhoeae (NG) that can provide accurate, rapid, and timely results are urgently needed so that patients can be treated at the time they visit a clinic. The Cepheid GeneXpert ${ }^{\circledR}$ (Xpert) CT/ NG assay is a rapid nucleic acid amplified test (NAAT) assay that can be performed in on-site laboratories. The assay detects DNA of CT and NG from female endocervical, patient-collected vaginal, and urine specimens, and in male urine from symptomatic and asymptomatic individuals.

Methods The Xpert test is a modular, cartridge-based, "walkaway" platform for testing which requires no manipulation from specimen loading until results are available (90 minutes). We compared results from the Xpert assay to results from two currently approved nucleic acid amplification assays (Aptima Combo2 and ProbeTec) in 1,722 females and 1,387 males. Results included a specimen adequacy control result and an amplification control result. The targets were two highly conserved, non-contiguous NG-unique chromosomal targets for NG and a chromosomal CT target.

Results Compared to patient-infected-status (PIS), the Xpert results for chlamydia demonstrated sensitivities for endocervical, vaginal, and urine samples of $97.4 \%, 98.7 \%$, and $97.6 \%$, respectively, in females and in male urine a sensitivity of $97.5 \%$. All specificity estimates were $\geq 99.4 \%$. Results for gonorrhoea demonstrated sensitivities for endocervical, vaginal, and urine samples of $100.0 \%$, $100.0 \%$, and $95.6 \%$, respectively, in females, with specificities > $99.9 \%$. In male urine, the sensitivity for gonorrhoea was $97.50 \%$ and the specificity was $99.9 \%$.

Conclusions The GeneXpert ${ }^{\circledR}$ System is a closed, self-contained, fully-integrated, automated platform for CT/NG testing that demonstrated excellent sensitivities and specificities in women and men. This relatively rapid short-turn-around-time test can provide results to guide treatment decisions before patients leave the clinical setting. Such rapid treatment could potentially improve chlamydia and gonorrhoea control efforts.

\section{P5.063 INFLUENCE OF TEMPERATURE, MEDIUM AND STORAGE DURATION ON CHLAMYDIA TRACHOMATIS DNA DETECTION BY POLYMERASE CHAIN REACTION}

doi:10.1136/sextrans-2013-051184.1107

1,2L van Dommelen, 1,2P F G Wolffs, ${ }^{1,2}$ F H van Tiel, ${ }^{3,1,2}$ N Dukers, ' $\mathrm{S}$ B Herngreen, ${ }^{1,2} \mathrm{C}$ A Bruggeman, ${ }^{3,1,2} \mathrm{C}$ J P A Hoebe. 'Maastricht University Medical Centre, Maastricht, The Netherlands; ${ }^{2}$ CAPHRI School of Public Health and Primary Care, Maastricht, The Netherlands; ${ }^{3}$ South Limburg Public Health Service, Geleen, The Netherlands

Background Chlamydia trachomatis $(\mathrm{Ct})$ is the most prevalent bacterial sexually transmitted microorganism worldwide. Many researchers conveniently use stored samples for $\mathrm{Ct}$ research. We assessed the impact of four different temperature conditions, six different types of medium and five increasing lengths of duration of storage, on Ct DNA detection.

Methods Phosphate buffered saline (PBS), 2-sucrose-phosphate (2-SP) medium, COBAS Amplicor medium and urine samples were spiked with the same amount of $\mathrm{Ct}$ serovar $\mathrm{D}$ elementary bodies and were stored in at room temperature (RT), $4^{\circ} \mathrm{C},-20^{\circ} \mathrm{C}$ and $-80^{\circ} \mathrm{C}$. Clinical $\mathrm{Ct}$ positive urine samples and $\mathrm{Ct}$ positive swabs in COBAS Amplicor medium were collected, pooled and stored at the same 4 temperatures. Samples (136 clinical and 287 spiked samples) were tested in triplicate on day 0 and subsequently after 1, 7,14 and 30 days of storage and two years hereafter for the presence of Ct DNA. Approximately 3000 plasmids were available per PCR reaction and each sample was thawed only once. Cycle thresholds were analysed using generalised linear models, controlling for repeated measurements.

Results Ct could be detected in all clinical samples and spiked media and cycle thresholds were stable over time with few exceptions. For Ct DNA detection in spiked COBAS Amplicor medium, cycle thresholds increased within the first month at $-20^{\circ} \mathrm{C}$ and $-80^{\circ} \mathrm{C}$ (both $\mathrm{p}<0.01$ ), but decreased in the samples frozen after two years of storage. In spiked urine and pooled clinical urine samples, the cycle threshold decreased within the first month $(p<0.01)$, including all but one $\left(4^{\circ} \mathrm{C}, \mathrm{p}=0.09\right)$ of the studied temperatures, but increased after two years in the frozen samples.

Conclusion Our results demonstrate that storage conditions and duration hardly affect Ct DNA detection by PCR in a negative manner, although frozen urine samples, stored for prolonged periods (more than two years), could become Ct negative.

\section{P5.064 PRELIMINARY ANALYTICAL EVALUATION OF ARTUS $®$ CT/NG, FOR SIMULTANEOUS DETECTION OF NEISSERIA GONORRHOEAE AND CHLAMYDIA TRACHOMATIS}

doi:10.1136/sextrans-2013-051184.1108

1,2S N Tabrizi, ${ }^{1} \mathrm{~J}$ Twin, ${ }^{3} \mathrm{M}$ Unemo, ${ }^{4} \mathrm{~A}$ Wiezer, ${ }^{5} \mathrm{~A}$ Limnios, ${ }^{5} \mathrm{M}$ Lahra, ${ }^{1,6} \mathrm{~S}$ Garland. ${ }^{1}$ The Royal Women's Hospital, Parkville, Australia; 'Department of Obstetrics and Gynaecology, University of Melbourne, Parkville, Australia; 'WHO Collaborating Centre for Gonorrhoea and other STIS, Department of Laboratory Medicine, Microbiology, Örebro University Hospital, Örebro, Sweden; ${ }^{4}$ OIAGEN Research and Development, Hamburg, Germany; ${ }^{5}$ WHO Collaborating Centre for STD, Microbiology Department, The Prince of UK Hospital, Randwick, Australia; ${ }^{62)}$ Department of Obstetrics and Gynaecology, University of Melbourne, Parkville, Australia

Introduction Currently there are a number of molecular amplification assays for detection of Neisseria gonorrhoeae and Chlamydia trachomatis. Prior to introduction of any new assay, it should be thoroughly evaluated for potential false positive and false negative results due to cross reaction with other species, and potential mutations and genetic exchanges with other closely related organisms. Artus ${ }^{\circledR}$ CT/NG is currently a research use only kit (with the fully automated version to be released later this year)for simultaneously detection of $C$. trachomatis and $N$. gonorrhoeae. We performed preliminary analytical evaluation of this assay.

Method This study evaluated the Artus ${ }^{\circledR}$ CT/NG assay with 290 characterised culture isolates obtained from the Neisseria Reference Laboratory at the World Health Organization Collaborating Centres for Sexually Transmitted Disease (STD) in Sydney and WHO Collaborating Centre for Gonorrhoea and other STIs in Örebro, Sweden. Strains included 148 N. gonorrhoeae isolates, 130 isolates of non-gonococcal Neisseria species, 12 isolates of other species closely related to Neisseria and 16 C. trachomatis strains of different serovars (including LGV and nvCT strains).

Results All C. trachomatis and N. gonorrhoeae isolates were detected. A detection sensitivity of 10 genome copies per reaction was obtained with all C. trachomatis serovars as well as a representative $N$. gonorrhoeae control strain. All 142 non-gonococcal isolates were negative on the assay. 